\title{
A New Flight Test Technique for Pilot Model Identification
}

\author{
Oliver Brieger ${ }^{1}$ and Daniel Ossmann ${ }^{2}$ \\ DLR - German Aerospace Center, Institute of Flight Systems, 85077 Manching, Germany \\ Markus Rüdinger ${ }^{3}$ \\ WTD 61 - German Bundeswehr Technical and Airworthiness Center for Aircraft, 85077 Manching, Germany \\ Matthias Heller ${ }^{4}$ \\ EADS Military Air Systems, 85077 Manching, Germany
}

\begin{abstract}
For today's highly augmented fighter aircraft, the aircraft dynamics are specifically tailored to provide Level 1 handling qualities over a wide regime of the service flight envelope. This requires a profound understanding of the human pilot to assure that stability margins of the airframe plus controller dynamics are sufficient to accommodate the additional pilot dynamics introduced into the system during closed loop tasks. Whereas the mathematical formulations of the airframe and controller dynamics are reasonably exact, the human pilot remains to be the most unpredictable element in the Pilot Vehicle System. In the past decades various pilot models have been developed in conjunction with analytical handling qualities and Pilot Involved Oscillations prediction criteria, mainly focusing on air-to-air tracking tasks. This paper focuses on the development of a novel flight test technique, which allows the identification of the pilot dynamics during air-to-surface aiming tasks. During an extensive flight test campaign, data was gathered and processed, using state of the art systemidentification techniques to derive a mathematical model of the human pilot during air-tosurface tracking tasks. Flight test and model-based data are compared with each other to support the validity of the developed pilot models.
\end{abstract}

\section{Nomenclature}

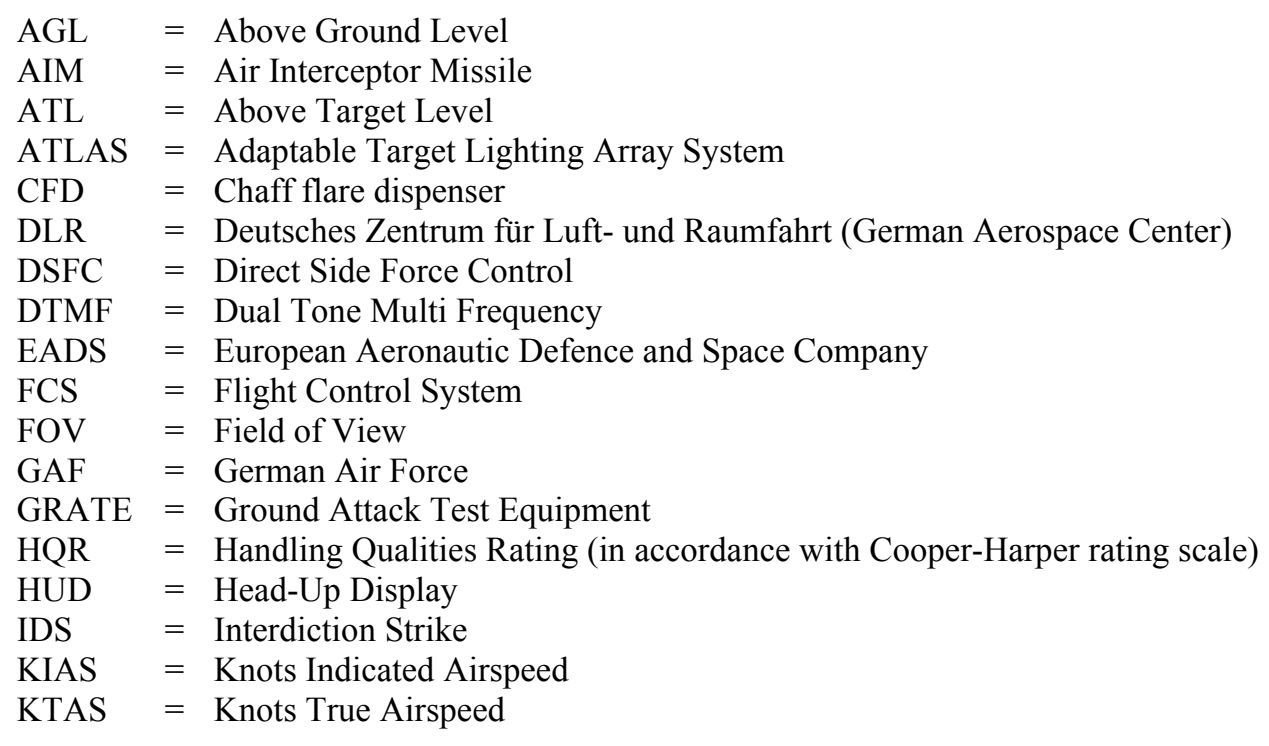

${ }^{1}$ Group Leader, Flight Test Branch, Ingolstadt/Manching Airbase, Member.

${ }^{2}$ Research Scientist, Flight Test Branch, Ingolstadt/Manching Airbase, Member.

${ }^{3}$ Test Pilot, German Air Force, Aircraft Evaluation Division, Ingolstadt/Manching Airbase.

${ }^{4}$ Expert Advisor Flight Dynamics, Flight Dynamics, PO Box 801229. 


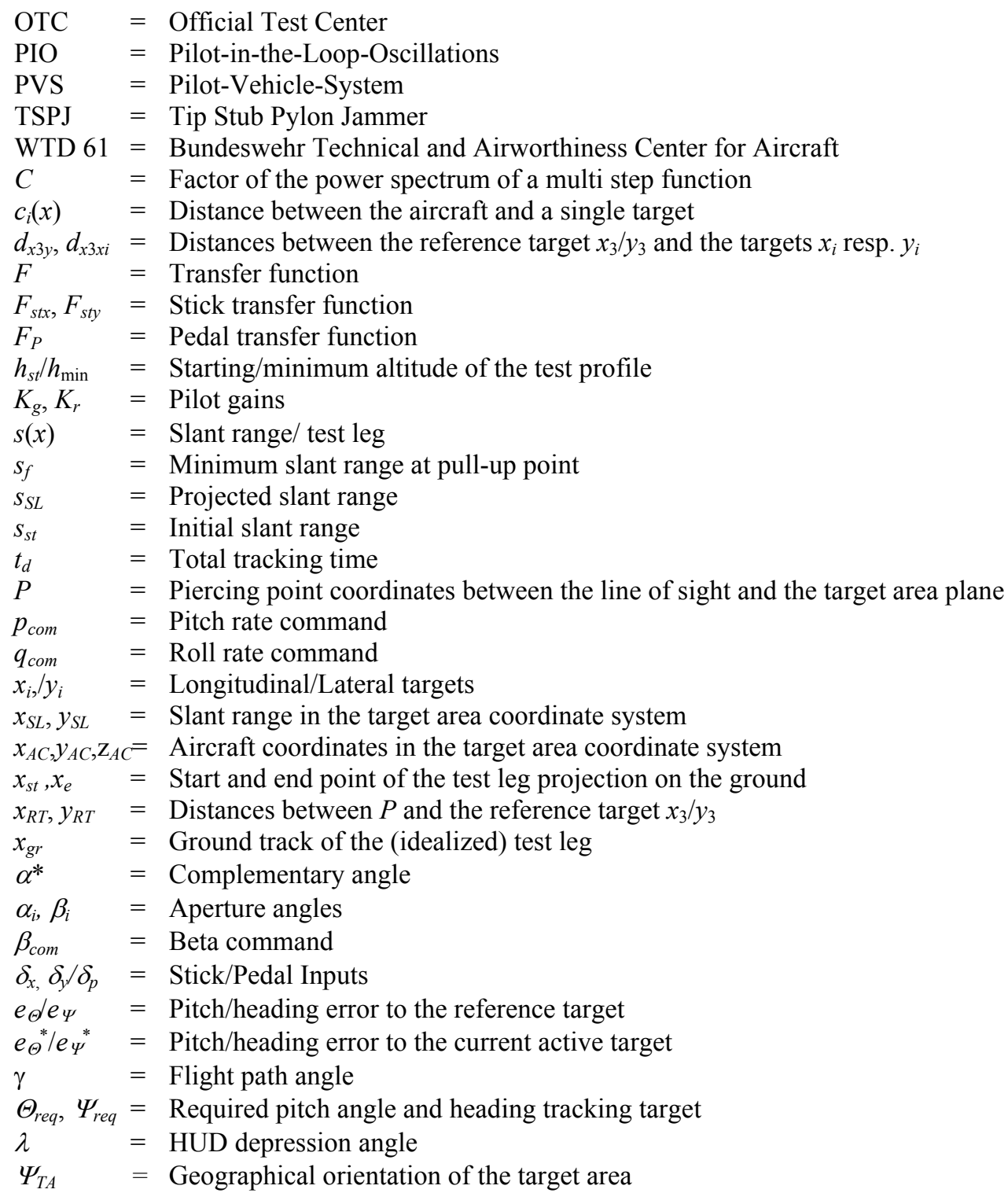

\section{Introduction}

$\mathrm{T}^{\mathrm{H}}$ HE first concepts of modeling the human pilot by means of gathered empirical data have been generated during World War II. After the first elementary applications in the years after the war, the development and utilization of pilot models has increased tremendously with the rapid development of complex flight control systems. Most pilot models are limited to one specific closed-loop task, as it is nearly impossible to derive a global mathematical description for the inherently nonlinear transitions in pilot behavior. Due to the unique human ability to learn, to adapt to varying circumstances very quickly using a great variety of human sensors and to establish a wide range of Pilot Vehicle System (PVS) organizations, the pilot is 'modifier' as well as 'operating entity' within the system. Even for one specific task (e.g. air-to-air refueling) the gross number of available sensing mechanisms to perceive and analyze perturbations in the environment and the strong influence of psychological factors such as stress, motivation or even fear in emergency situations have great impact on human behavioral patterns. Another factor is the individual mental constitution, which may affect pilot actions in various ways. All these characteristics make it extremely difficult to develop an appropriate mathematical model of the pilot, suitable for the assessment of handling qualities.

Nevertheless, numerous mathematical descriptions of man-machine-interaction have been derived in the past decades and constitute the basis for a large number of handling qualities criteria, which are essential for the evaluation of modern, highly augmented aircraft. For the description of the overall PVS the application of modern control theory is appropriate. As shown in the example given in Fig.1 the pilot generates the necessary command action to 
minimize the system error between the actual and desired aircraft pitch attitude $\Theta$ by processing the perceived information.

Early approaches are based on more or less complex analytical descriptions of the pilot by using transfer and describing function techniques to model the human pilot as an active, dynamic control element. In the course of model development, the pilot block in Fig. 1 has been enhanced to include multiple, complex blocks, comprising sensor channels, higher brain structures, the neuromuscular system and biomechanics. Every one of these blocks proc-

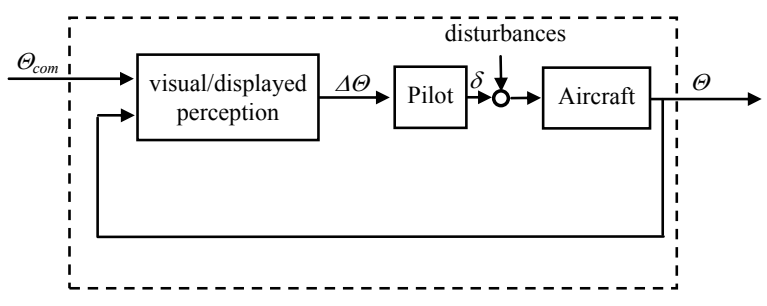

Figure 1. Man-machine-control-loop (closedloop pitch tracking model) esses and advances signal information and can be translated into a transfer/describing function. In other words, the pilot model has grown from a very simple transfer element to a more complex structural model, with numerous feedback loops.

The assumption that the human operator can be described by a controller which estimates the state of the controlled system and develops a control strategy to attain a defined performance index has led to the development of the optimal control model, using optimal control theory. These models are rather complicated, but are capable of describing the human behavior in many different situations. Other fields of research focus on biomechanical descriptions of the human pilot, analyzing acceleration influences on the pilot's arm as a passive, biodynamic element which may unintentionally induce control inputs. Such investigations are important to validate highly augmented aircraft with respect to their sensitivity to acceleration coupling effects like role ratcheting. ${ }^{1}$ Today, engineers attempt to model the human operator's behavior using novel concepts based on fuzzy logic, neuronal networks or Petri nets. ${ }^{1}$ These go as far as replicating the human decision making and learning process. All these approaches have their legitimacy. Nevertheless, many state of the art handling qualities criteria, employed in the control law design process of modern fighter aircraft, make use of classical pilot models, based on linear/quasi-linear transfer functions optimized for one specific closed-loop task. The vast majority of these models, mostly derived from flight test data, have been developed for air-to-air tracking tasks. The work described herein, however, focuses on the identification of a linear, structural pilot model, applicable to air-to-surface gun attacks. In order to achieve this aim, already existing structural models are used and adapted to the air-to-surface tracking task by means of system identification. The main difference in terms of pilot behavioral patterns is that during air-to-air tracking a combination of previewing and compensatory behavior can be observed. This is related to the significant lead compensation the pilot can introduce into the closed-loop system when initially acquiring the target, anticipating the projected target trajectory before transitioning to predominantly compensatory behavior during fine tracking. For the air-to-surface tracking task described herein, the previewing element is minimized, requiring the pilot to employ a purely compensatory control strategy.

The associated flight tests have made extensive use of the newly developed Ground Attack Test Equipment II (GRATE II), which is based on a ground deployed variable target system GRATE jointly developed by the German Aerospace Center (DLR) and the Bundeswehr Technical and Airworthiness Center for Aircraft (WTD 61) in the 1980 s, originally designed to assess the handling qualities of various combat aircraft during gun strafing. ${ }^{4}$ Since fuselage pointing is an extremely demanding task, requiring precise aircraft control, a method was sought which facilitates safe, repeatable, precise, high gain tasks during simulated ground attacks in a realistic, operationally relevant environment. The system employs an array of lighted targets which are placed at specified positions on the ground. During a prolonged dive attack the target lights are illuminated in a predefined sequence. The pilot's task consists of expeditiously acquiring and precisely tracking the respective lighted target, which appear to be selected randomly, with an aircraft fixed reference until the next target is illuminated. The objective is to force the pilot to react continuously utilizing a high gain compensatory piloting technique, while minimizing any lead compensation. Consequently, the closed-loop PVS is excited over a wide frequency range. This method was applied with great success in 1984 during the Direct Side Force Control (DSFC)-Alpha-Jet Program and proved to be very effective and reliable in detecting handling qualities deficiencies. In 1987 NASA's Dryden Flight Research Center developed a functional equivalent of the system known as Adaptable Target Lighting Array System (ATLAS). ${ }^{6}$ Apart from the evaluation of handling qualities this system is also suitable to investigate pilot dynamics, since it provides a precisely defined input signal into the PVS. Provided that the mathematical descriptions of the airframe and controller dynamics are accurate, the derivation of a suitable pilot model becomes viable. This second application of the GRATE-System has been considered in the past but never realized. Unfortunately, both the original GRATE and ATLAS-Systems have been lost over the past years, so that the development of a new, more sophisticated GRATE II-System became inevitable. A detailed system description is given in the following section. The determination of the target array geome- 
try, the target sequencing, defining the exciting function of the PVS, and the aircraft trajectory are of utmost importance. They define the quality of the gathered data and consequently the validity of the derived pilot model. A comprehensive description of the target sequences with respect to an optimized spectral density over a wide frequency range and a detailed description of the flown attack profiles are given. To account for varying pilot perception and technique three test pilots were involved in the flight test campaign and a total of 50 test runs have been performed. The paper concludes with a portrayal of the system identification method used to process the gathered flight test data using DLR's system identification tool FITLAB (see Ref. 9, 10) to derive decoupled, linear pilot models for the longitudinal and lateral-directional motions which replicate pilot behavior during air-to-surface tracking. Time histories generated with model data are then compared with actual flight test data to evaluate model fidelity.

\section{Flight Test Preparation}

\section{A. Description of the GRATE II System}

The main objective during the development of GRATE II was to design an effective system, which offers maximum flexibility with respect to transportability, time-efficient assembly and simple reconfiguration capability while still fulfilling flight test relevant requirements, such as response characteristics and good visibility throughout the simulated gun attacks. The latter is achieved by using high intensity approach lights as commonly used for runway approach lighting systems. These can be precisely adjusted in elevation angle and azimuth, to focus the beams on the projected approach flight path. The reconfiguration aspect has been realized by using wireless technol-

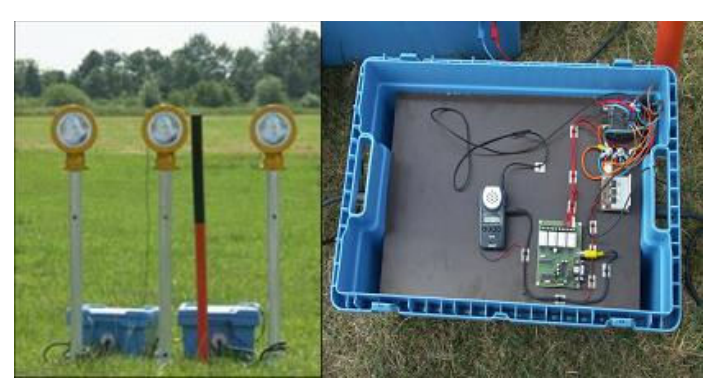

Figure 2. GRATE II target unit ogy, enabling a quick adaptation to varying flight test objectives (longitudinal or lateral-directional handling qualities evaluations, system identification). Every target unit con-
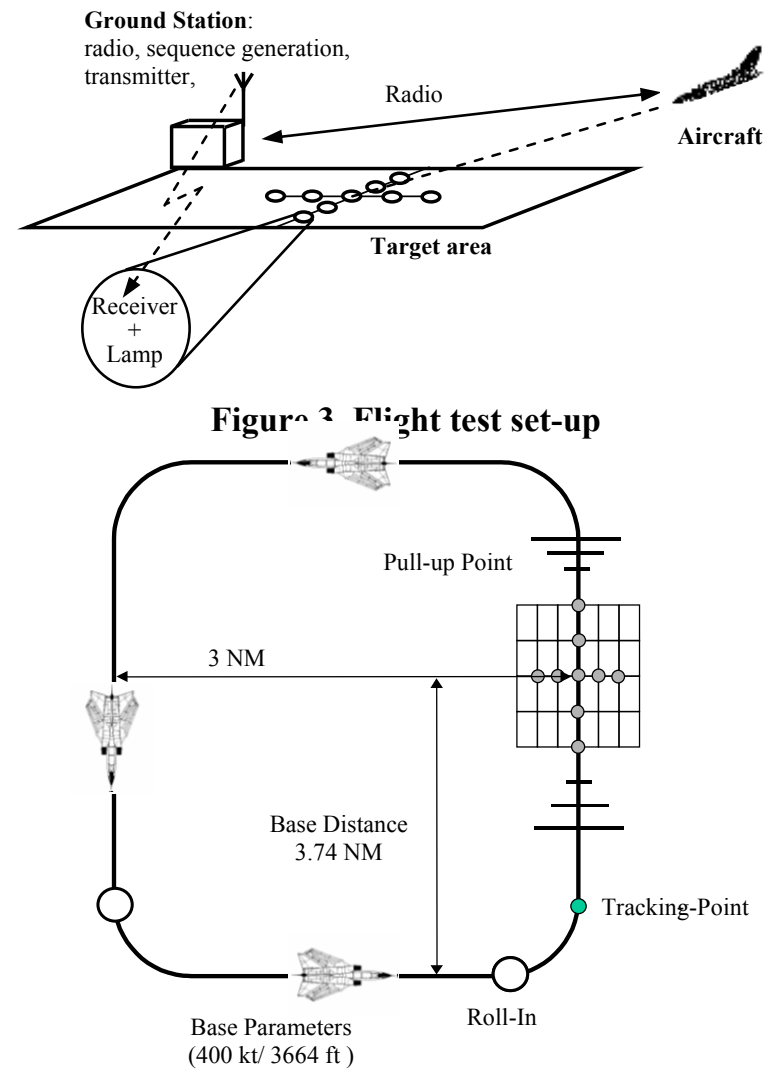

Figure 4. Target pattern sists of three approach lights, each mounted on a ground spike with an independent power supply (Fig. 2).

To provide as much flexibility as possible the target sequences are generated using a graphical user interface based on Matlab ${ }^{\circledR}$, installed on a laptop. To avoid interferences with other frequencies, which may disturb the transmission from the laptop to the targets and disrupt test runs, unique DTMF (Dual Tone Multi Frequency)frequencies are used to command the switching of receiver relays and lamps. The DTMF audio signals generated with Matlab ${ }^{\circledR}$ are sent to the radio transmitter via the sound output of the laptop. The transmitter routes the audio signal to the receivers of all target units. Since every unit has been assigned an individual frequency, only those lights of the associated unit are illuminated as long as the signal is transmitted. Figure 3 illustrates the basic flight test set-up.

The pilot flies a race track pattern around the target area as depicted in Fig. 4. Once established on the final inbound run-in leg to the target area the test sequence is initiated on the pilot's call. The pilot then acquires and tracks the illuminated targets using an aircraft-fixed reference. The target sequences have been designed to either excite the longitudinal motion, the lateraldirectional motion, or a combination of all three axes.

\section{B. Definition of the Target Area Geometry and An- gular Relationships}

As described earlier, a fundamental prerequisite for the accuracy of the subsequent pilot model identification 
process is the distinctive excitation of the PVS, governed by the target positions on the ground, the illumination sequence as well as the aircraft position and attitude relative to the target area. The consecutive illumination of the targets during the test runs and the resulting changes in the pilot's line of sight constitutes the input signal into the PVS. Consequently, the first step is to define the target positions on the ground followed by the determination of the angular correlation between the individual targets and the aircraft. Due to the extensive research conducted during GRATE development in the 1980s substantial experience with respect to an effective test execution was readily available, expanding into the design and enhancements of the GRATE II system. Therefore, a similar aircraft approach geometry with comparable flight parameters for gun strafing was adopted:

- flight path angle $\gamma$ of $\sim-10$ deg

- constant airspeed of $400 \mathrm{KTAS}$

- $18 \mathrm{~s}-20 \mathrm{~s}$ evaluation time per run

- a minimum height of $500 \mathrm{ft}$ ATL (Above Target Level)

- constant progression of aperture angles throughout the test run

The test aircraft used for this flight test campaign was a GAF Panavia Tornado operated by WTD 61 (Fig. 5). A validated linear model of the aircraft was subsequently used for the identification of the pilot dynamics based on the

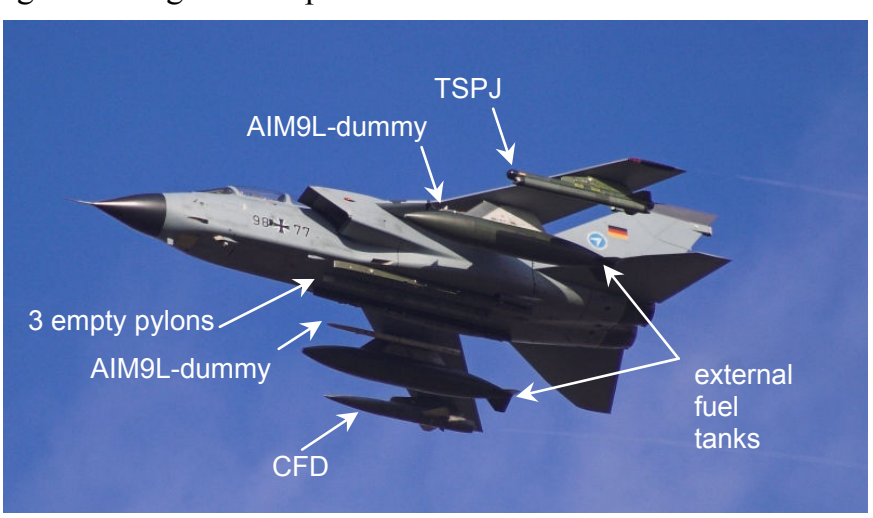

Figure 5. Test aircraft (courtesy of Mr. Christian Röttinger) gathered flight test data. Parameters of the attack trajectory, i.e. minimum recovery altitude, altitude loss during recovery, safety margins, etc. have been determined using a high fidelity non-linear simulation model prior to flight testing.

Initially, a decoupled analysis of the longitudinal and lateral-directional motion is envisaged. Future research will also cover fully coupled investigations. Therefore, a target configuration is required, which allows a separate excitation of the lateral-directional and longitudinal axes but also provides the capability to stimulate all axes simultaneously. Additionally, this should be facilitated without the need to reconfigure the target area while testing is in progress. To fulfill these requirements a cross-shaped configuration comprising nine target units was found to be the most suitable. The target sequences used to excite the PVS consist of six successive target illuminations in either the

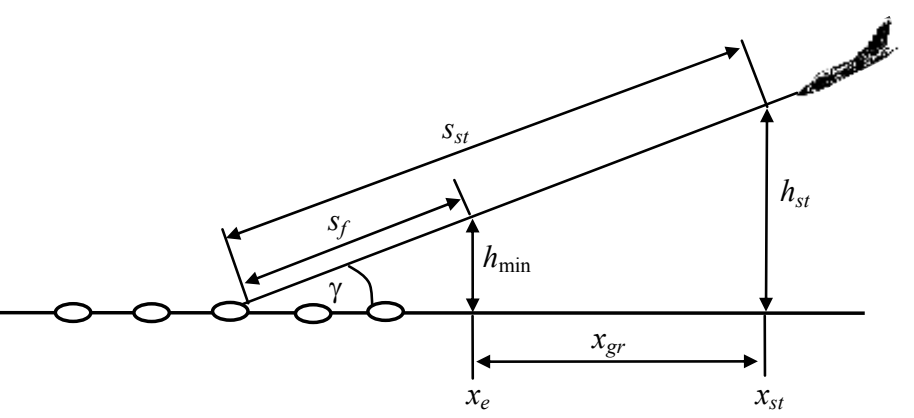

Fig. 6: xz-plane of approach trajectory longitudinal, lateral-directional or in a combination of these axes. The illumination duration for one target is varied between $2.25 \mathrm{~s}$ and $3 \mathrm{~s}$ but is held constant throughout a test run. The reasoning behind these values will be explained later on. This leads to a total tracking time $t_{d}$ between $13.5 \mathrm{~s}$ and $18 \mathrm{~s}$. Figure 6 depicts the general attack scheme. Assuming calm atmospheric conditions and neglecting the barometric pressure difference between the tracking point at $h_{s t}$ and the pull-up point at $h_{\min }$ the initial slant range equates to

$$
s_{s t}=s\left(x_{s t}\right)=s_{f}+\left(h_{s t}-h_{\min }\right) / \underbrace{\sin (\gamma)}_{s\left(x_{e}\right)}
$$

Since the research objective is the identification of linear pilot models, perturbations need to be kept small. Target angle variations should therefore not exceed $1 \mathrm{deg}$, as described in Ref. 7. While the aircraft approaches the target area the aperture angles between the targets increase. Nevertheless, the angle variations induced by the target sequence should approximately remain the same to generate homogenous data throughout the run. Therefore, also a minimum angle variation of $0.4 \mathrm{deg}$ is defined in Ref. 7. Bearing these constraints in mind, the calculation of the target spacing for the longitudinal and lateral-directional axis is performed independently. Target $x_{3}$, as depicted in Fig. 7 and 8 is used as reference point and origin of the target array coordinate system since it constitutes the pilot's initial aiming point before the target sequence is initiated. 


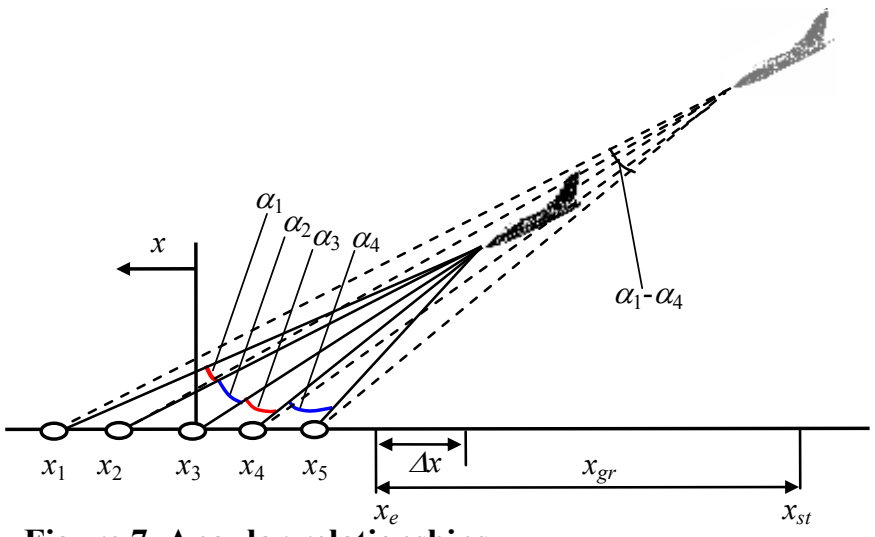

Figure 7. Angular relationships

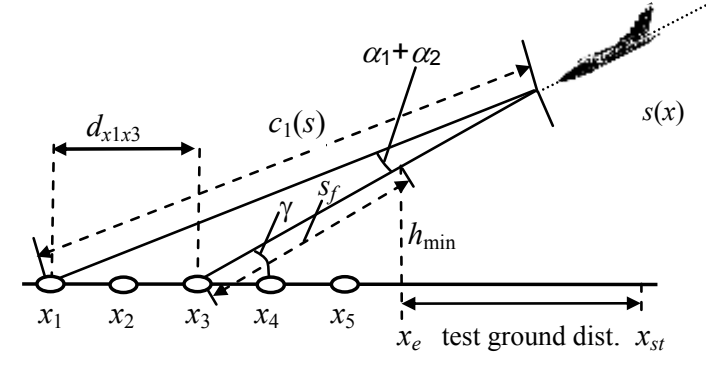

Figure 8. Characteristic distances

Figure 7 and 8 also show the target configuration for the excitation of the longitudinal axis. The aperture angles $\alpha_{1}$ $\alpha_{4}$ between adjacent targets increase as the aircraft approaches. As depicted in Fig. 7 an equidistant spacing between the target units would lead to $\alpha_{4}>\alpha_{3}>\alpha_{2}>\alpha_{1}$ along the entire test section. While initially the angle variations would be rather small, they would significantly increase as the aircraft progresses along the test leg. This effect needs to be attenuated and a nearly uniform progression of the aperture angles $\alpha_{1}-\alpha_{4}$ has to be assured. Consequently, the spacing between the target units has to be defined in such a way, that $\alpha_{3}$ equals $1 \mathrm{deg}$ at the minimum slant range $s\left(x_{e}\right)$ to meet the defined requirements stated above. This, however, leads to an $\alpha_{4}\left(x_{\mathrm{e}}\right)$ greater than $1 \mathrm{deg}$. But since the last target change is triggered earlier at $s\left(x_{e}-\Delta x\right)$, where $\alpha_{4}$ is still within limits, this is considered acceptable. By observing these constraints the distance $d_{x 3 \times 4}$ between the reference target $x_{3}$ and target $x_{4}$ can be determined by applying the sinus theorem, based on the geometric interrelationships described in Fig. 9.

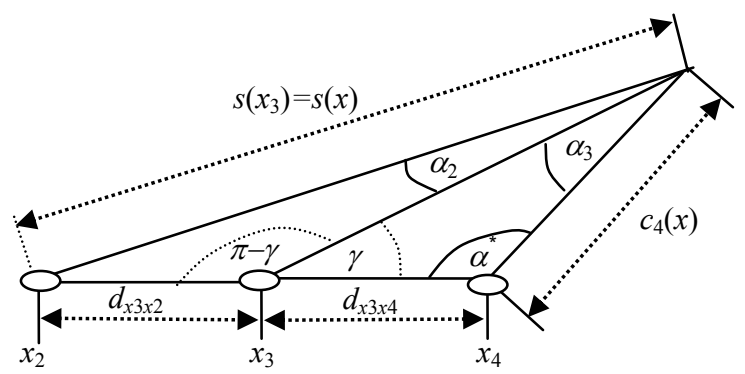

Figure 9. Slant range geometry

$$
d_{x 3 \times 4}=s(x) \frac{\sin \left(\alpha_{3}\right)}{\sin \left(\alpha^{*}\right)}
$$

By inserting the corresponding values at the pull-up point $x_{e}$ on the attack trajectory into Eq. (2) the distance $d_{x 3 x 4}$ can be calculated as follows:

$$
\begin{aligned}
& d_{x 3 \times 4}=s(x) \frac{\sin \left(\alpha_{3}(x)\right)}{\sin \left(\alpha^{*}(x)\right)}=s_{f} \frac{\sin \left(\alpha_{3}\left(x_{e}\right)\right)}{\sin \left(\pi-\left(|\gamma|+\alpha_{3}\left(x_{e}\right)\right)\right)} \\
& \text { with } \quad \alpha_{3}\left(x_{e}\right)=1 \mathrm{deg},|\gamma|=10 \mathrm{deg}, s_{f}=1225 \mathrm{~m}
\end{aligned}
$$

This gives a distance $d_{x 3 \times 4}$ of $112 \mathrm{~m}$. Subsequently, the variation of $\alpha_{3}$ as a function of the aircraft ground track $x$ can be determined by applying the laws of sine and cosine:

$$
\begin{aligned}
& \alpha_{3}(x)=\arcsin \left[\frac{d_{x 3 x 4}}{s_{x 4}(x)} \sin (\gamma)\right] \\
& \text { with } s_{x 4}(x)=\sqrt{d_{x 3 x 4}^{2}+s^{2}(x)-2 d_{x 3 x 4} s(x) \cos (\gamma)}
\end{aligned}
$$

The remaining aperture angles $\alpha_{4}, \alpha_{2}$ and $\alpha_{1}$ can then be expressed accordingly: 


$$
\begin{aligned}
& \alpha_{4}(x)=\arcsin \left[\frac{d_{x 3 x 5}}{s_{x 5}(x)} \sin (\gamma)\right]-\alpha_{3}(s) \\
& \text { with } s_{x 5}(x)=\sqrt{d_{x 3 x 5}^{2}+s^{2}(x)-2 d_{x 3 x 5} s(x) \cos (\gamma)} \\
& \alpha_{2}(x)=\arcsin \left[\frac{d_{x 3 x 2}}{s_{x 2}(x)} \sin (\pi-\gamma)\right] \\
& \text { with } s_{x 2}(x)=\sqrt{d_{x 3 x 2}^{2}+s^{2}(x)-2 d_{x 3 x 2} s(x) \cos (\pi-\gamma)} \\
& \alpha_{1}(x)=\arcsin \left[\frac{d_{x 3 x 1}}{s_{x 1}(x)} \sin (\pi-\gamma)\right]-\alpha_{2}(x) \\
& \text { with } s_{x 1}(x)=\sqrt{d_{x 3 x 1}^{2}+s^{2}(x)-2 d_{x 3 x 1} s(x) \cos (\pi-\gamma)}
\end{aligned}
$$

The distances between the remaining targets and the reference target $x_{3}, d_{x 3 x 5}, d_{x 3 x 2}$, and $d_{x 3 x 1}$ are determined by means of a numerical optimization method to obtain an approximately homogenous progression of the aperture angles as a function of the covered ground distance. This is to ensure comparable angle alterations throughout the test run. The employed algorithm minimizes the error between the enclosed reference area defined by the integral of Eq. (4) and the integrals of Eq. (6), (8) and (10) as described in Eq. (12):

$$
e\left(d_{x_{3} x_{m}}\right)=\left|\int_{x_{s t}}^{x_{e}} \alpha_{3}(x) d x-\int_{x_{s t}}^{x_{e}} \alpha_{m}\left(x, d_{x_{3} x_{m}}\right) d x\right| m=1,2,4
$$

The resulting distances are listed in Tab. 1 below.

\begin{tabular}{|l|l|}
\hline$d_{x 3 \times 1}$ & $258 \mathrm{~m}$ \\
\hline$d_{x 3 \times 2}$ & $123 \mathrm{~m}$ \\
\hline$d_{x 3 \times 4}$ & $112 \mathrm{~m}$ \\
\hline$d_{x 3 \times 5}$ & $214 \mathrm{~m}$ \\
\hline
\end{tabular}

Table 1. Distances between the reference target $x_{3}$ and $x_{i}$

Figure 10 depicts the aperture angle progression of $\alpha_{1}-\alpha_{4}$ as a function of the covered ground distance $x$. It is evident that initially the angle values are relatively small, around 0.2 deg. To warrant angle alterations between $0.4 \mathrm{deg}$ and $1 \mathrm{deg}$, initially only every other target is illuminated while towards the end of the run adjacent targets are activated. An exemplary sequence for the longitudinal axis is $x_{1} \rightarrow x_{4} \rightarrow$ $x_{2} \rightarrow x_{4} \rightarrow x_{3} \rightarrow x_{2}$ (please refer to Fig. 7).

To finalize the definition of the target cross configuration the distances between the targets for the excitation of the lateral-directional motion (excitation along the $y$-axis of the target array) as illustrated in Fig. 11 have to be determined. These are spaced with identical increments due to symmetry aspects. Since again the small purtubation approach applies, the following assumption can be made: $\tan (\beta)=\beta$ for $\beta<<\pi$. With the requirement $\beta\left(x_{\mathrm{e}}\right)=1 \mathrm{deg}$ the lateral distances between target units can then be calculated,

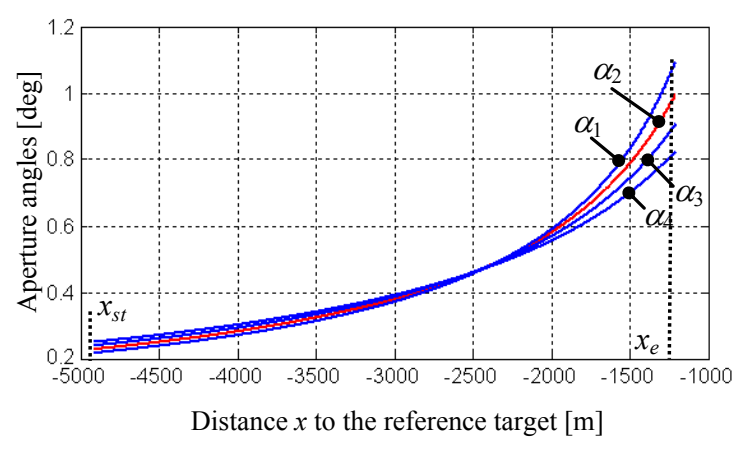

Figure 10. Aperture angle progression as a function of covered ground distance

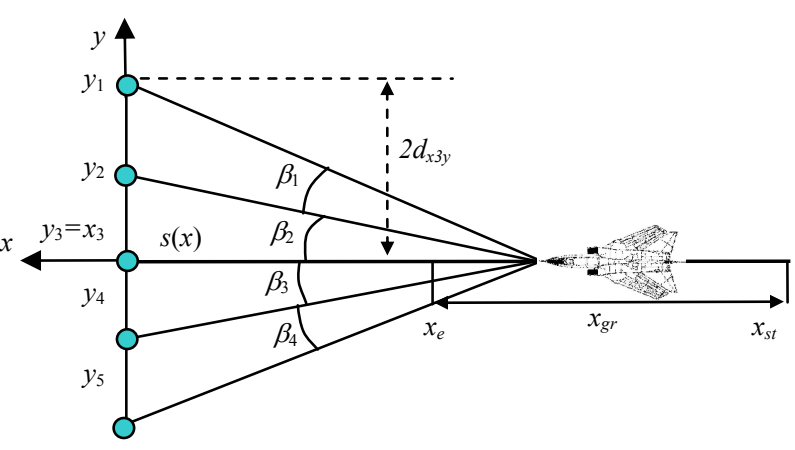

Figure 11. $x y$-plane of the approach trajectory which in turn enables the determination of the 
progression of the angles $\beta_{1^{-}} \beta_{4}$ along the test leg $s(x)$. By solving Eq. (14) the equidistant lateral spacing $d_{x 3 y}$ equates to $21.5 \mathrm{~m}$

$$
\begin{aligned}
& \beta_{i}(s)=\arctan \left(\frac{d_{x 3 y}}{s(x)}\right) \\
& \text { with } d_{x 3 y}= \pm \frac{s(x)}{\sin (\pi-\gamma-\pi / 180)} \sin (\pi / 180)
\end{aligned}
$$

An excitation sequence for the lateral-directional axes is for example is $y_{3} \rightarrow y_{6} \rightarrow y_{4} \rightarrow y_{3} \rightarrow y_{7} \rightarrow y_{5}$ (please refer to Fig. 11).

The target array layout has now been defined. Albeit a fully coupled investigation has not yet been conducted for the initial pilot model identification process, which focuses on the development of decoupled longitudinal and lateraldirectional models, the resulting angular relationships for an excitation of the PVS in multiple axes can be determined as follows. The cross array allows numerous angle variations by alternately illuminating targets in the $\mathrm{x}-$ and $\mathrm{y}$-axis as depicted in Fig. 12, showing all but the mirror-symmterical target combinations. In the following, the derivation for one exemplary set of angles will be presented, the remaining sets are obtained accordingly. An illustration of the angular relationships for the calculation of $\delta_{x 1 y 1}(x)$, the enclosed angle between target unit $x_{1}$ and $y_{1}$ is depicted in Fig. 13. The slant range $s_{y 1}(x)$ between the aircraft and target unit $y_{1}$ can be determined with the given distance $d_{x 3 y}$ (Eq. (14)). The slant range $s(x)=s_{x 3}(x)=s_{y 3}(x)$ to the reference target in the origin of the target array is calculated by:

$$
s_{y 1}(x)=\sqrt{s(x)^{2}+4 d_{y 3 y}^{2}}
$$

For the determination of the angle $\delta_{x 1 y 1}(x)$ the ground distance $d_{x 1 y 1}$ is required. This is easily derived by:

$$
d_{x 1 y 1}=\sqrt{4 d_{x 3 y}^{2}+d_{y 3 x 1}^{2}}
$$

Finally, the angle $\delta_{x 1 y 1}(x)$ can be expressed by applying the law of cosines and substituting Eq. (11), (15) and (16):

$$
\delta_{x 1 y 1}(x)=\arccos \frac{s_{y 1}^{2}(x)+s_{x 1}^{2}(x)-d_{x 1 x 2}^{2}}{2 s_{y 1}(x) s_{x 1}(x)}
$$

The remaining angles $\delta_{x i y i}(x)$ are calculated accordingly, by inserting the slant ranges $s_{x i}(x)$ and $s_{y i}(x)$ for $i=1: 9$. The postulated limits between $0.4 \mathrm{deg}$ and $1 \mathrm{deg}$ are also valid for the combined excitations. In Fig. 14 the resulting angle progressions, with the color code used to highlight target changes in Fig. 12, are plotted as a function of covered ground distance. It shows the defined angular limits of $0.4 \mathrm{deg}$ and $1 \mathrm{deg}$ and the position of the last possible target alteration. This is defined by the transition from the $5^{\text {th }}$ to the $6^{\text {th }}$ target prior to pull-up and the longest illumination interval of $3 \mathrm{~s}$. Fig. 14 therefore defines the possible target combinations within the given limits. To warrant angle alterations between $0.4 \mathrm{deg}$ and $1 \mathrm{deg}$, initially the consecutive illumination of the outer targets in both axes is favorable (target alterations defined by the red and black curves) while towards the end of the run the inner targets have to be activated (target alterations defined by the blue curves); also refer to Fig. 12 for conclusiveness.

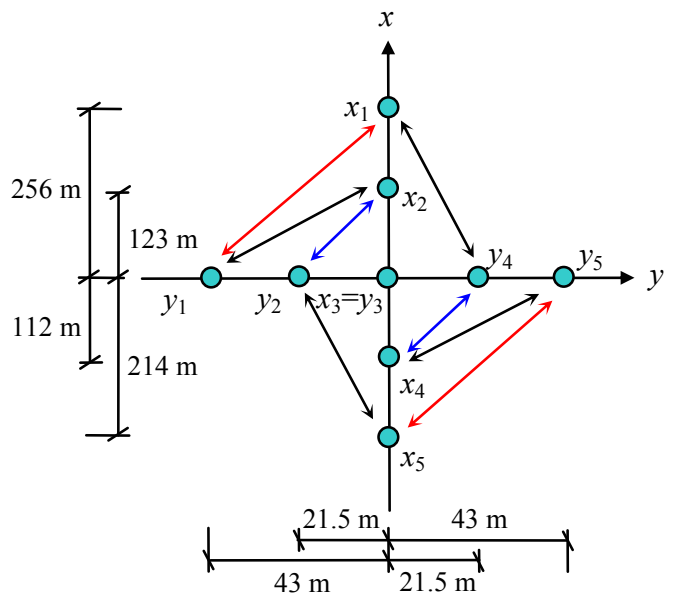

Figure 12. Possible target combinations for combined inputs

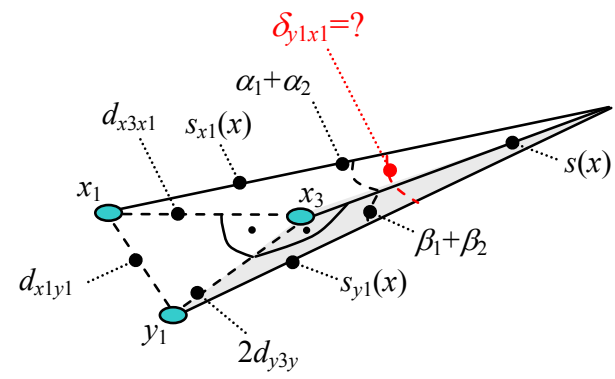

Figure 13. Angular relationships for $\delta_{x 1 y 1}(x)$

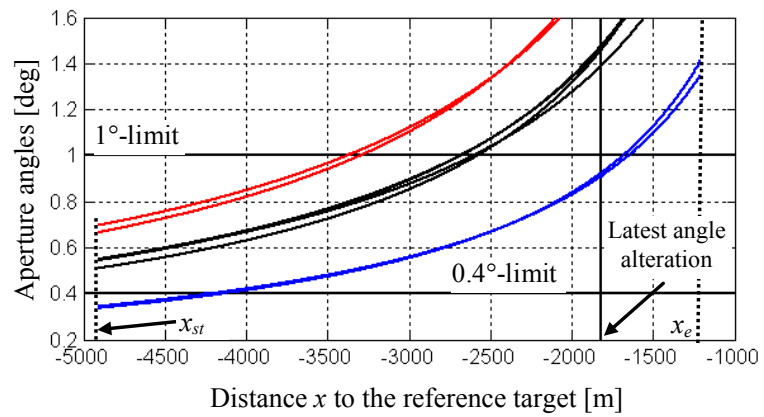

Figure 14. Progression of combined aperture angles 


\section{Target Sequences Generation}

In order to identify the pilot's transfer behavior within the PVS, a sufficient excitation over a wide frequency range is required. ${ }^{1}$ The input signal into the PVS during a GRATE II tracking task is generated by the continuous variation of the line of sight in pitch and in yaw (in $\alpha_{i}$ and $\beta_{i}$ ) between the aircraft and the varying ground targets. An exemplary curve progression of the changing line of sight for an excitation in the longitudinal axis with a time increment of $\Delta t=3 \mathrm{~s}$ and a trimmed airspeed of 400 KTAS is depicted in Fig. 15.

The varying line of sight, defined by the illumination sequence, constitutes the input signal into the PVS and can be approximated by a multi-step function $r(t)$, as depicted in Fig. 16. The power spectrum of this function $r(t)$ can be expressed by:

$$
\begin{aligned}
& \frac{|R(\omega)|^{2}}{T}=\underbrace{2 \Delta t \frac{1-\cos (\omega \Delta t)}{(\omega \Delta t)^{2}}}_{C} \ldots \\
& \underbrace{\left[\frac{1}{N} \sum_{j=1}^{N} v_{j}^{2}+\frac{2}{N} \sum_{j=1}^{N-1} \cos (i \omega \Delta t) \sum_{k=1}^{N-1} v_{j+k} v_{k}\right]}_{P}
\end{aligned}
$$

The contributions of the factors $C, P$ and the resulting amplitude spectrum, which can be determined by computing the square root of Eq. (18), are plotted in Fig. 17 for $\Delta t=3 \mathrm{~s}$. The amplitude spectrum for $C$ clearly decreases with increasing frequency. The second factor $P$ in Eq. (18) is periodic with $T=2 \pi / \Delta t$. Therefore, it is not possible to generate signals with a constant power spectral density - a decreasing amplitude cannot be avoided. Nevertheless, the main aim is to define the target excitation sequence in such a manner, that a nearly uniform spectrum can be achieved over a wide frequency range. Fig. 18 shows the root of the dominant factor $\mathrm{C}$ as a function of varying time intervals $\Delta t$ and frequency $\omega$. For small time intervals the spectrum is rather constant. As $\Delta t$ increases a shift of the first zero and an increase in maximum amplitude towards $\omega=0$ at the expense of the local maximum at $\omega=2 \pi$ can be observed. Therefore, target sequence time intervals should be made as small as possible to guarantee uniform amplitude and power spectra but since PVS-bandwidth is limited, they cannot be reduced without any constraints. Reasonable time intervals have been calculated during extensive research conducted in Ref. 7 and lie between $2.25 \mathrm{~s}$ and $3 \mathrm{~s}$. These were found to be acceptable for pilots, considering human and system reaction time. By varying the time interval $\Delta t$ in the given range it is possible to reduce the sweeping influence of the zeros by shifting their position. Since for the subsequent identification process multiple time slices of runs with different

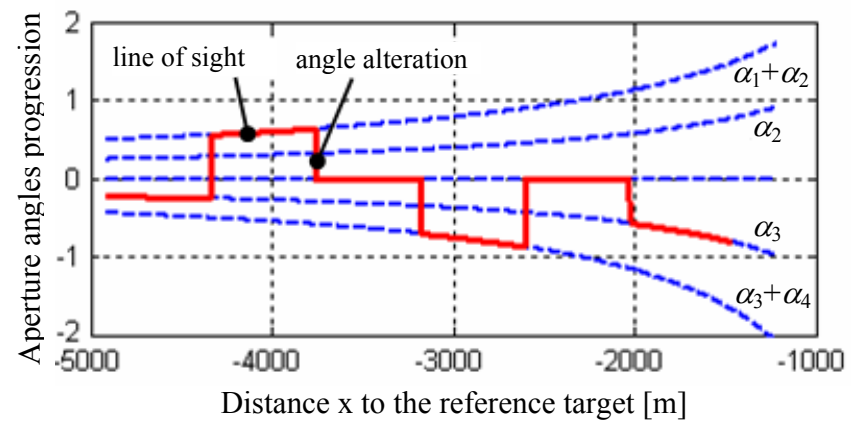

Figure 15. Example of a PVS-input signal in pitch

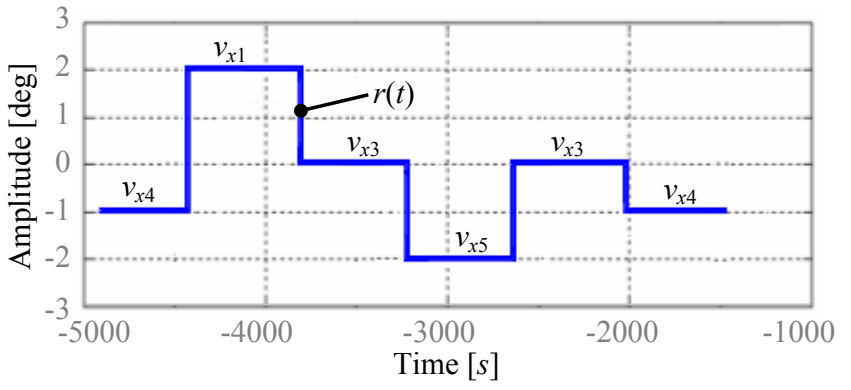

Figure 16. Multi-step approximation of the PVS input signal

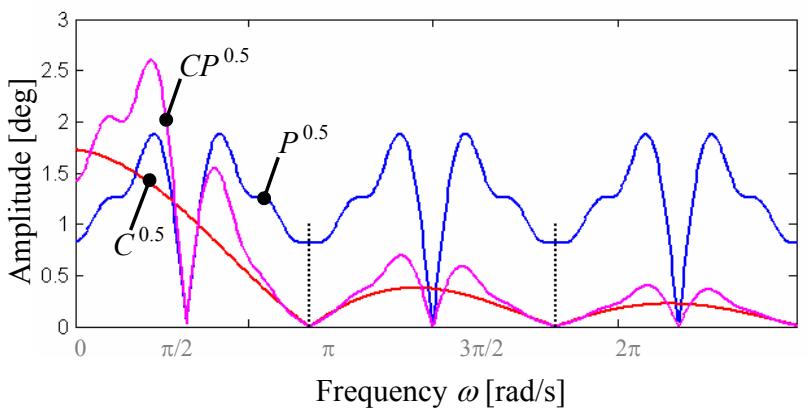

Figure 17. Amplitude spectra for a PVS input signal $(\Delta t=3 \mathrm{~s})$

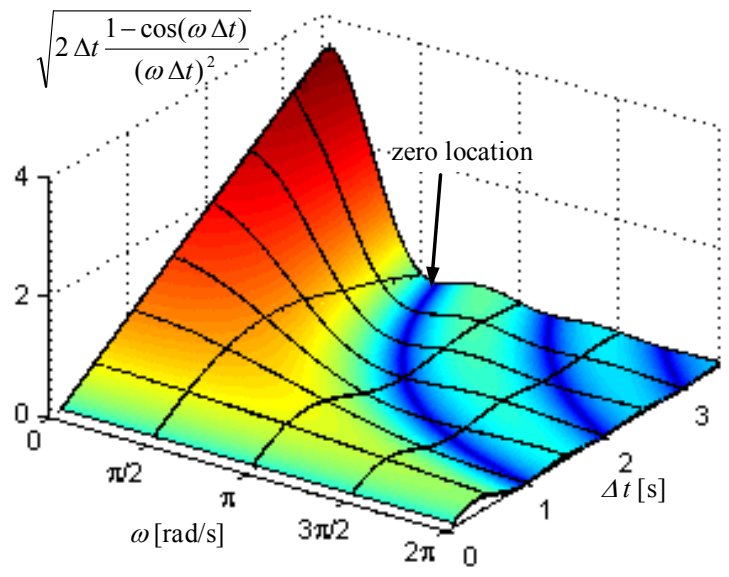

Figure 18. Amplitude density of $C^{0.5}$ as a function of $\Delta t$ and $\omega$ 
target time intervals are used, the resulting model is valid for a broad frequency range without encountering shortcomings in signal quality evoked by zeros in the amplitude spectrum. In summary, the PVS-input signals for the excitation of individual and multiple axes are obtained by means of a complex optimization algorithm which considers the following constraints:

- Variation of $\Delta t$ between $2.25 \mathrm{~s}$ and $3 \mathrm{~s}(0.15 \mathrm{~s}$ increments $)$

- Uniform amplitude spectrum

- Angle alterations limited to $0.4-1.0 \mathrm{deg}$

- Longitudinal transitions to the final $6^{\text {th }}$ target are always 'nose up' for flight safety reasons

\section{Determination of System Inherent Time Delays}

An extensive study regarding system inherent time lags has been conducted, to determine rise and decay times of the 200 Watt approach lights, since this significantly affects pilot reaction time. Specifically the period of time from the instant the DTMF-signal is transmitted to the respective target unit until the light intensity has reached a level that the pilot becomes aware of the target change, is essential. Equally important is the determination of the decay time, since the decay of the light intensity of the high intensity lights is much slower than the onset, which can lead to a delayed shift of attention, because the pilot may still focus on the deactivated target, while the new target is already visible. A complex test setup was devised to measure the target lamp performance and to derive a mathematical approximation. The setup included an infra-red LED to measure light intensity and measurement devices, recording signal generation, signal transmission and relay switching. The decay behavior is approximated by a $7^{\text {th }}$ order polynomial, while the onset behavior is approximated by multiple functions, including linear and $5^{\text {th }}$-order approximations, as depicted in Fig. 19.

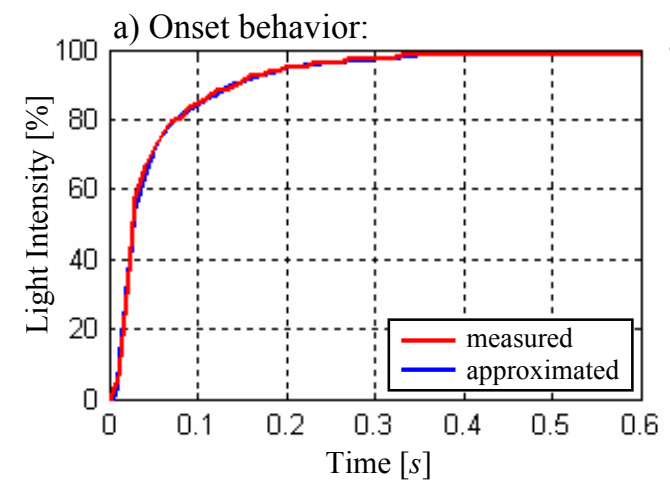

b) Decay behavior:

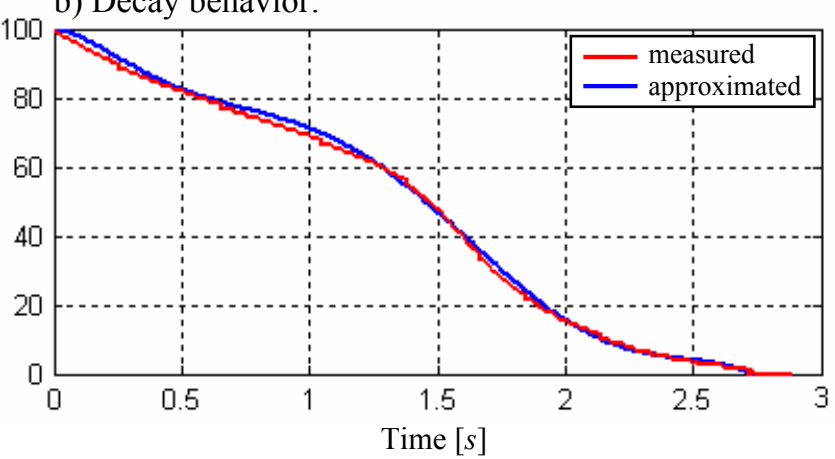

Figure 19. Mesured and approximated onset and decay beavior

Since the activation of the new target and the deactivation of the old target occur simultaneously, it is difficult to determine, to which stimulus the pilot will react to. For the determination of the inherent system time delay the worst case is assumed, where the pilot may continue to track the already deactivated target, due to the much slower decay in light intensity, before becoming aware that a new target has been activated. Earlier flight tests using GRATE II have shown that an average $0.5 \mathrm{sec}$ system inherent time delay is a good first estimate and may be changed as a result of the system identification process.

\section{Flight Test Execution}

\section{A. Test Aircraft}

The test bed for this test was a GAF Tornado PA200 Interdiction Strike (IDS) Version with variable wing sweep geometry. The external aircraft configuration consisted of two external 1500 ltrs fuel tanks on the inboard wing stations, a chaff/flare dispenser (CFD) and a jamming pod (TSPJ) on each outboard wing station. Additionally, two Air Interceptor Missiles (AIM-9L) dummies were carried on both side wall stations (see Fig.5). This test configuration is a standard aircraft configuration, as well as mission representative (typical egress phase during a combat sortie with all bombs delivered). Total aircraft weight varied between approximately $47.000 \mathrm{lbs}$ on the first run to 31.000 lbs on the last run.

For post flight data analysis the onboard test recording equipment was used as well as the standard video system, which recorded the HUD-video and the right multi-function display in the rear cockpit. The sample rate for all flight relevant parameters ranged from $8 \mathrm{~Hz}-16 \mathrm{~Hz}$. From the three possible wing sweep settings $(25 \mathrm{deg}, 45 \mathrm{deg}$ and 67 
deg) the 45 deg setting was used for all test runs, since this is the most likely configuration in the low altitude regime. Furthermore, the manual selectable slats were set to 'in'.

The flight control system of the aircraft is a complete irreversible fly-by-wire system, with pitch-/ roll-rate and sideslip angle command. Pitch is controlled via the symmetric deflection of the all movable taileron, whereas rollcontrol is achieved by the asymmetric deflections of the taileron surfaces assisted by one pair of spoilers on each wing. Yaw is commanded via a classical rudder.

\section{B. Test Pattern Description}

In order to maximize the possible test-runs per sortie a typical race track pattern as depicted in Fig. 4, was established known from typical air-to-ground bombing ranges. All legs of the pattern were planned as to arrive on the final run-in with the correct flight parameters considering aircraft weight and configuration. Based on the chosen flight path angle, the required evaluation time and the planned airspeed on the final test-leg, the entire geometry and the respective altitudes were calculated backwards. Different roll-in techniques were taken into account as well as approximately $2.3 \mathrm{~s}$ to establish and stabilize the parameters after rolling out on final. Furthermore, a minimum altitude over the test area was set to $500 \mathrm{ft}$ AGL and an altitude loss during dive recovery of $250 \mathrm{ft}$ ( $4 \mathrm{~g}$ recovery) considered.

\section{Run-In Parameters}

The flight-test parameters on the evaluation leg of the pattern were chosen based on operational relevance for typical air-to-ground tasks while still fulfilling all test requirements. The flight path angle was therefore set to $-10 \mathrm{deg}$, which is generally the minimum angle for a typical airto-ground gun attack. A steeper approach would have been more advantageous in a tactical environment; however, this would have required a longer final run-in leg due to the higher recovery altitude after the dive, possibly impairing the visibility of

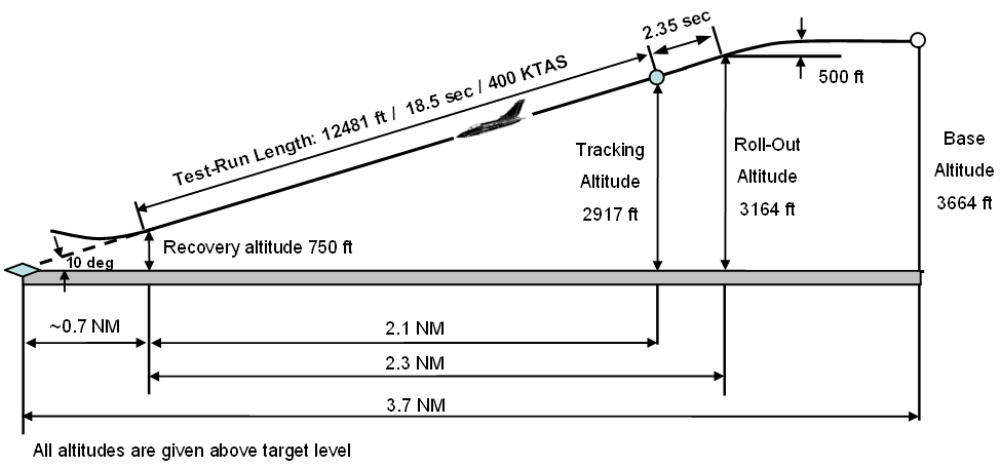

Figure 20. Test profile the target lights under all lighting conditions. Target airspeed was governed by the same trade-off between operational and test requirements and was set to $400 \mathrm{KTAS}$, which was equal to approximately $385 \mathrm{KIAS}$ considering the atmospheric conditions during the tests. In Fig. 20 a cross section of the final run-in with all relevant descriptions is depicted.

\section{Piloting Requirements}

All aircrew were test-crews from the German Official Test Center (OTC) at Manching (qualified test pilots with at least 1400 flying hours) and rated on this aircraft type. A total of three flights with three different pilots were conducted. Each sortie consisted of a familiarization phase and a dedicated test phase. The familiarization phase consisted of two runs to become acquainted with the test pattern and equipment; these two runs were also used to confirm the pre-planned test pattern and optimize the alignment maneuver onto the final test leg. Afterwards 12-18 dedicated test-runs were conducted.

The combined task for the pilot during the evaluation consisted of an aggressive acquisition and precise tracking of the respective illuminated target. As aiming device, the reversionary bombing sight, projected into the HUD-FOV was used; this was calibrated once and not changed during all three test flights.

\section{System Identification and Pilot Model Development}

\section{A. System Identification}

For the identification of a pilot model for air-to-surface tracking tasks, system identification software developed at DLR Braunschweig called FITLAB is utilized. ${ }^{10}$ The basic concept of system identification is to derive a mathematical description of a dynamic system from the observed (measured) response $z$ to a known input $u$, by determining the underlying parameters $p$ which govern system behavior (see Fig. 21). For the objective described herein two steps need to be performed: First, the correct model structure (system state function $f$ and observation function $g$ ) describing the transfer behavior between $u$ and $z$ needs to be defined. Secondly, the system parameters $p$ have to be 
estimated by utilizing system identification methods which use the measured input and output data. The identification of the system parameters $p$ in the time domain, as applied here, is based on a maximum likelihood estimation employing numerical algorithms such as Gauss-Newton. A detailed description of the theoretical background of system identification methods would go beyond the scope of this paper, for a comprehensive, detailed treatment please refer to Ref. 9. The described approach of model development and parameter estimation is an iterative process, possibly requiring changes and adaptations within the model structure to achieve better identification re-

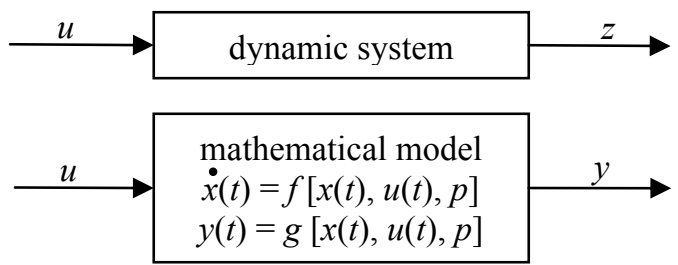

Figure 21. Basic scheme of the SysId process results.

\section{B. Tracking Error Function}

The output signals $z$ for the identification of the human pilot are the measured stickand pedal deflections during the tracking task. The input signals $u$ into the pilot model are the visual errors $e_{\Theta}$ and $e_{\psi}$, the difference between the required and the actual pitch attitude and heading angles. These are calculated from the measured Euler angles and the aircraft position as indicated in Fig. 21.

The idealized progressions of the aperture angles $\alpha_{i}$ and $\beta_{i}$, depending on the idealized

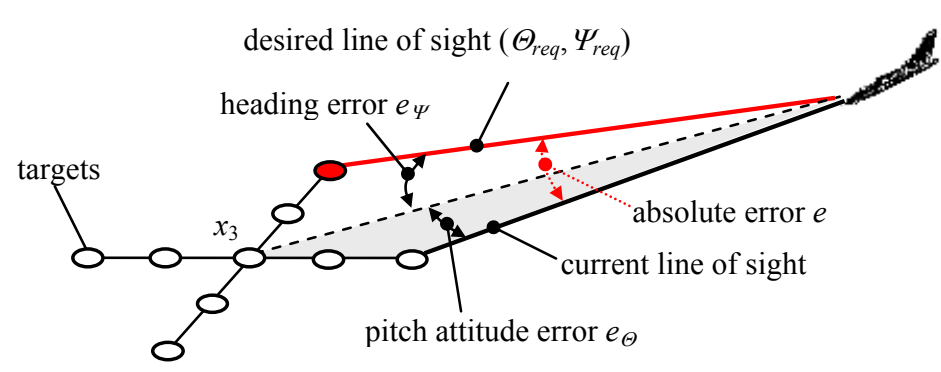

Figure 21. Visual tracking error position of the aircraft on the test leg have been presented in section II-B. It is obvious that the pilot cannot fly precisely on the idealized test leg, due to atmospheric disturbances or variations in the execution of the race track pattern. Hence, the true tracking error has to be determined by making use of the gathered flight test data. First, the coordinates need to be determined, where the line of sight from the design eye point through the HUD-fixed aiming device impinges the ground in the target array coordinate system, defined in Fig. 12. The idealized test leg and the $x$ axis of the target area have a westerly orientation with a true heading of $\Psi_{T A}=264.3 \mathrm{deg}$. Depending on the aircraft height (AGL) $z_{A C}$, pitch attitude angle $\Theta$, and the depression angle of the $\operatorname{HUD}(\lambda=0.085$ mils $)$, the projection of the line of sight onto the ground, coming from the aircraft can be determined as follows (also see Fig. 22a):

$$
s_{S L}=z_{A C} \tan (\pi / 2-|\Theta|-\lambda)
$$

With the distance $s_{S L}$ and the true heading $\Psi_{\text {of }}$ the test leg given, the piercing point $P$ with the target area plane can be expressed in polar coordinates within the aircraft fixed coordinate system (also see Fig. 22b):

$$
P\left(s_{A C}, \psi\right)=s_{S L} e^{\psi}
$$

The projection of the actual test leg on the ground can now be transformed into Cartesian coordinates:

$$
\begin{aligned}
& x_{S L}=s_{S L} \cos \left(\Psi-\Psi_{T A}\right) \\
& y_{S L}=s_{S L} \sin \left(\Psi-\Psi_{D A}\right)
\end{aligned}
$$

For the determination of the tracking errors it is necessary to transform the above coordinates into the target area coordinate system with $x_{3} / y_{3}$ as origin.

$$
\begin{aligned}
& x_{R T}=x_{A C}-x_{S L} \\
& y_{R T}=y_{A C}-y_{S L}
\end{aligned}
$$

a) Side view:

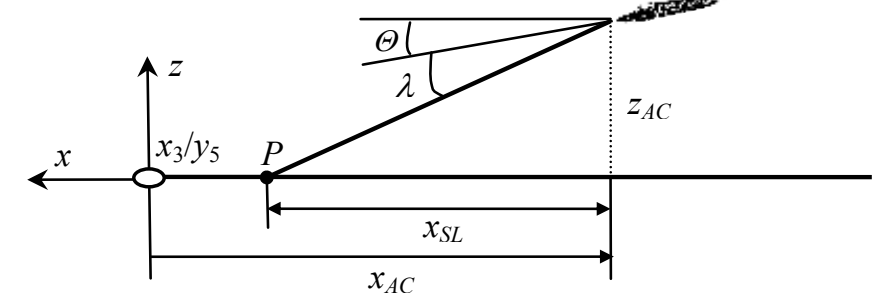

b) Top view:

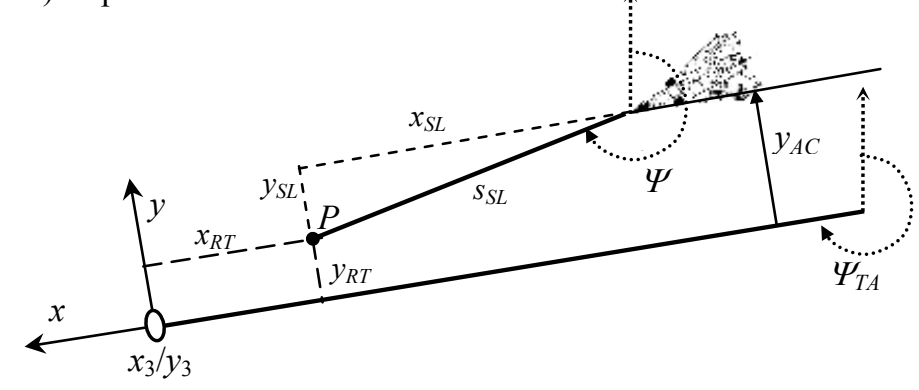

Figure 22. Projected slant range $s_{S L}$ and intersection point $P$ 
Significant deviations from the planned attack heading of $\Psi_{T A}=264.3 \mathrm{deg}$ would have the effect, that the target alterations in pitch would inevitably lead to undesired coupling between the longitudinal and lateral-directional axes. As described in Ref. 8, the GRATE II flight tests have shown that deviations in heading angle do not exceed \pm 2 deg, having, therefore, a negligible effect on the tracking error function. The longitudinal tracking error $e_{\Theta}$ can then be expressed as:

$e_{\Theta}=v-[\pi / 2-(|\Theta|+\lambda)]$

with

$v=\arctan \left(\frac{s_{S L}+x_{R T}}{z_{A C}}\right)=\arctan \left(\frac{x_{A C}}{z_{A C}}\right)$

The lateral tracking error is given by:

$e_{\Psi}=\arctan \left(\frac{y_{R T}}{s_{S L}+x_{R T}}\right)=\arctan \left(\frac{y_{A C}}{x_{A C}}\right)$

So far, only the tracking errors with respect to the reference target unit $x_{3} / y_{3}$ have been determined. The tracking error regarding the currently illuminated target unit can easily be calculated by correcting, $x_{R T}$ and $y_{R T}$ with the given distances $d_{x 3 x i}$ and $d_{x 3 y}$ (determined earlier in section II-B).

In Fig. 24 time histories of the angular errors $e_{\Theta}$ and $e_{\Psi}$ for a combined excitation sequence are shown. Clearly visible are the changes in tracking error magnitude when switching from one target to another every 3 sec. After each target alteration the pilots tries to minimize the longitudinal and lateral tracking error.

a) Longitudinal error $e_{\Theta}$ :

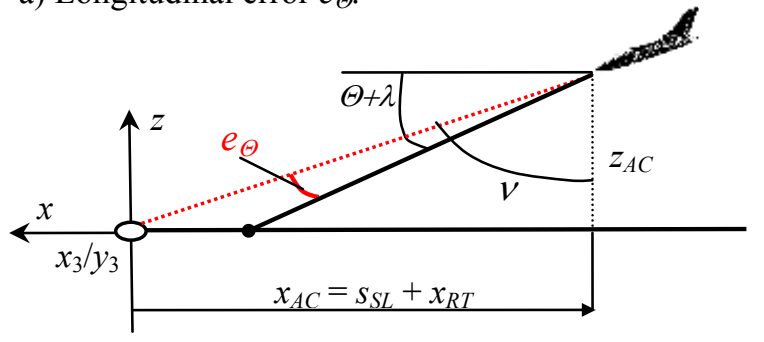

b) Lateral error $e_{\psi}$ :

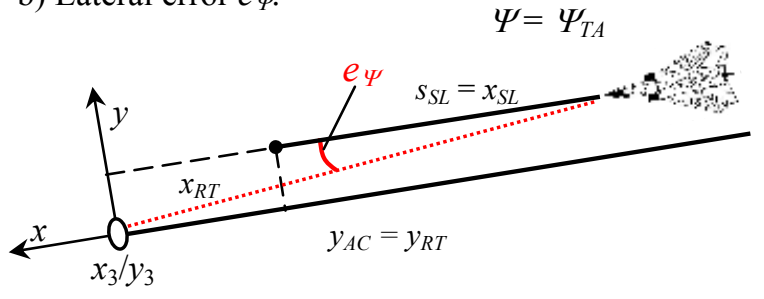

Figure 23. Geometrical depiction of the angular errors
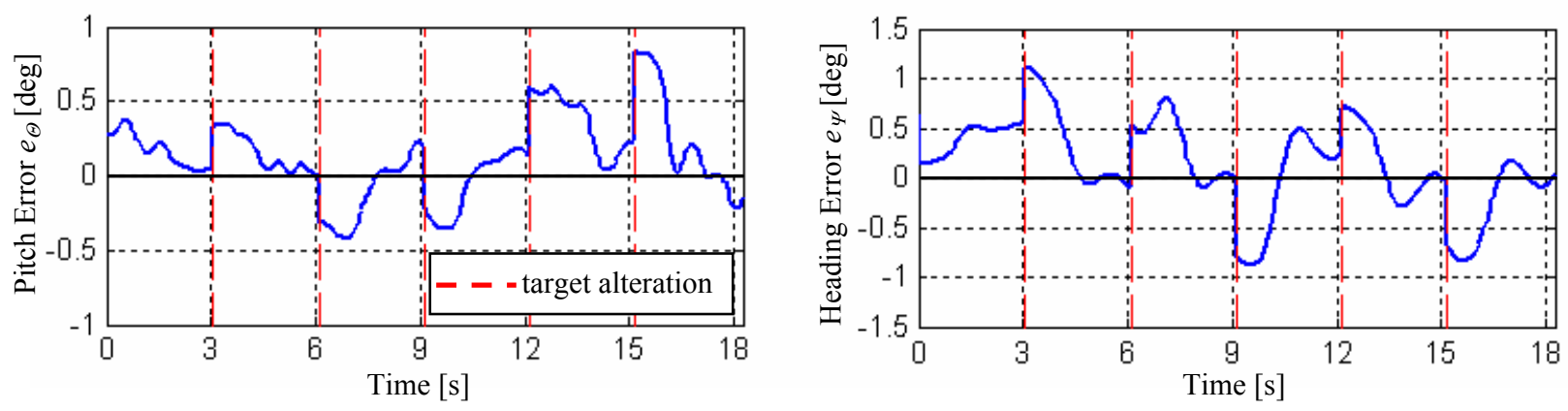

Figure 24. Example of $e_{\Theta}$ and $e_{\psi}$ during a combined test run

\section{System Identification}

The analysis of the flight test data has shown that during a solely longitudinal or lateral-directional excitation of the PVS the pilot focuses on the currently excited axis, neglecting any error in the other axes as long as it is small. Hence, if a single-axis excitation is given, the identification process can be focused on this axis alone. An elementary block diagram is depicted in Fig. 25. The linear pilot model is developed in a step-by-step approach, starting with the longitudinal axis, followed by the more complex lateral-directional axes. It is based on the crossover model theory described in Ref. 2 and the structural model developed in Ref. 3. 


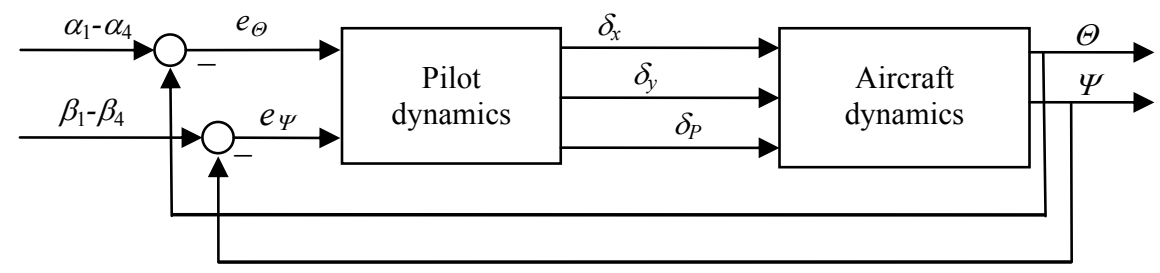

Figure 25. In- and outputs of the pilot, minimizing both errors by the use of all three control devises

\section{Longitudinal Axis}

During an air-to-surface tracking task with an excitation of the longitudinal axis, the pilot attempts to minimize the error $e_{\Theta}$. Hence, the pilot closes the loop for pitch attitude control $\Theta$ to determine the current error with respect to the illuminated target unit. For highly augmented aircraft, the pilot's stick input can be converted into various command signals, depending on the control law design. In the case of the Tornado the pilot commands the desired pitch rate $q_{\text {com }}$ by longitudinal stick deflections. Since the pilot uses the pitch attitude $\Theta$ to control the aircraft during the tracking task, the transfer behavior $q_{c o m} \rightarrow \Theta$ is characterized by a single integral with a proportional element $(\mathrm{K} / \mathrm{s})$ (see Ref. 2), when considering the short term dynamics only (which can be assumed for this application since target sequencing is very quick). The derived pilot model is shown in Fig. 26.

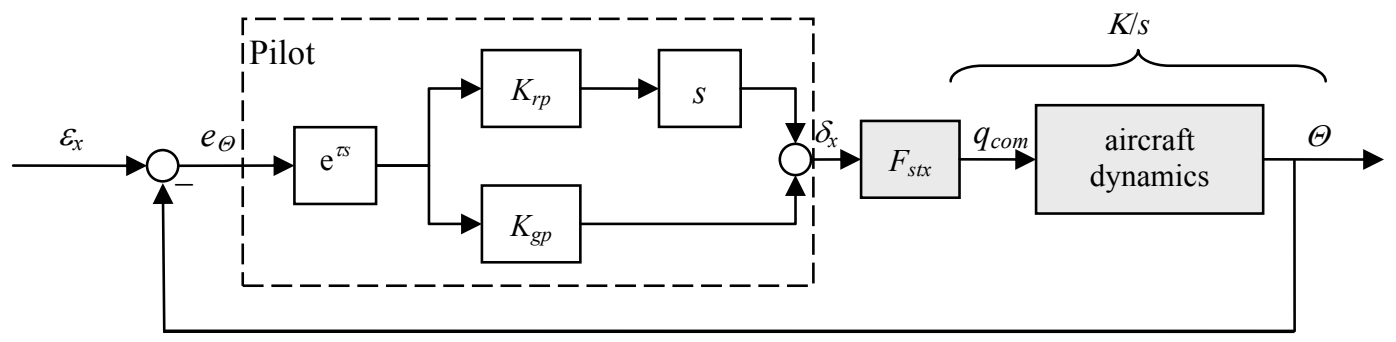

Figure 26. Pilot model structure for the longitudinal axis

The applicable state equation is easily derived and can be stated as follows:

$$
\delta_{x}=\left(K_{g p} e_{\Theta}+K_{r p} \dot{e}_{\Theta}\right) e^{2 s}
$$

The model takes the following aspects into account: the error signal $e_{\Theta}$ is delayed by $e^{\tau s}\left(1^{\text {st }}\right.$ order Padeapproximation), to account for the processing time required by the pilot to recognize target changes and generate appropriate stick inputs $\delta_{x}$. The total time delay is assumed to be $0.8 \mathrm{sec}, 0.5 \mathrm{sec}$ attributed to the GRATE II system as described in section II-D and $0.3 \mathrm{sec}$ of pilot delay, as commonly used in many handling qualities criteria. When acting on the error signal the pilot uses two sources of information; firstly, the absolute difference between desired and commanded pitch attitude represented by the $K_{g p}$ proportional path, and secondly, the perceived rate of change of the error, described by the proportional and derivative path. Hence, the $K / s$ transfer behavior of the open loop PVS described by the crossover model, as proposed in Ref. 2, is confirmed, however augmented with an additional rate command path.

Figure 27 shows the results of the system identification process for the longitudinal axis. An average time delay has been

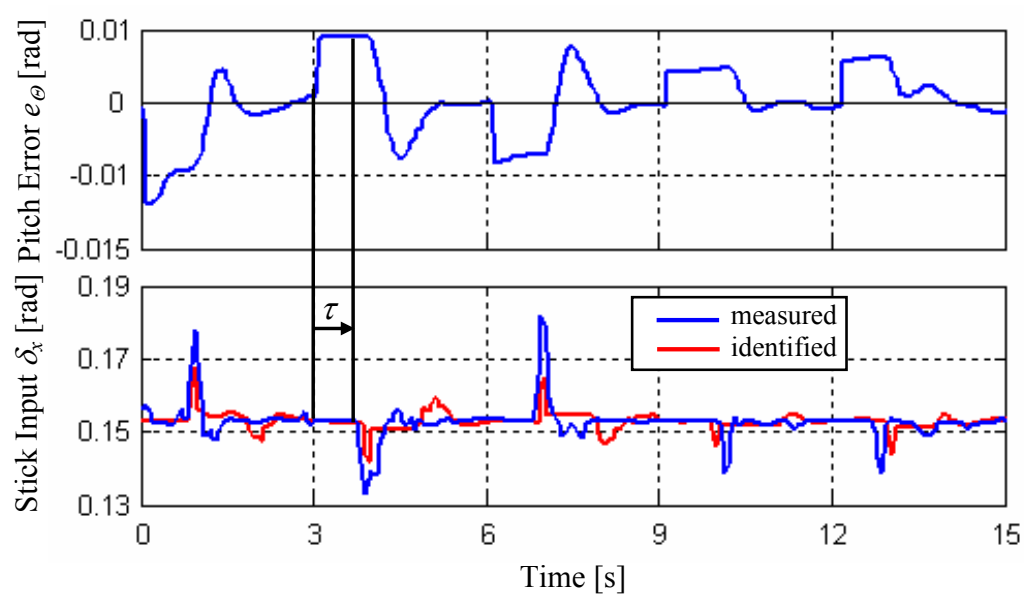

Figure 27. Longitudinal pilot dynamics 
estimated, which correlates with the initial approximation quite accurately. Deviations in stick amplitude may be associated with non-linear pilot dynamics, non-linear elements in the flight control system, and shortcomings in the pilot model, such as for instance neuro-muscular dynamics that have not been considered.

\section{- Lateral-directional Axes}

Contrary to the longitudinal excitation, the pilot controls two axes using rudder and aileron to minimize the error $e_{\Psi}$ during a pure lateral excitation. Similarly to the pitch axis, a (roll-) rate command system is used to control the aircraft. In the directional axis sideslip angle $\beta$ is directly controlled using the rudder, as a result inducing a change in heading angle $\Psi$. Flight test data analysis has shown that all pilots mainly used the rudder to place the aiming sight on the target, supported by only small roll inputs. The reason is that the aircraft response is very precise when mainly working with the pedals, combined roll-yaw inputs, when attempting to realign the aircraft on a straight trajectory towards the target led to overshoots and a significantly higher workload. The pilot model for this case is shown in Fig. 28.

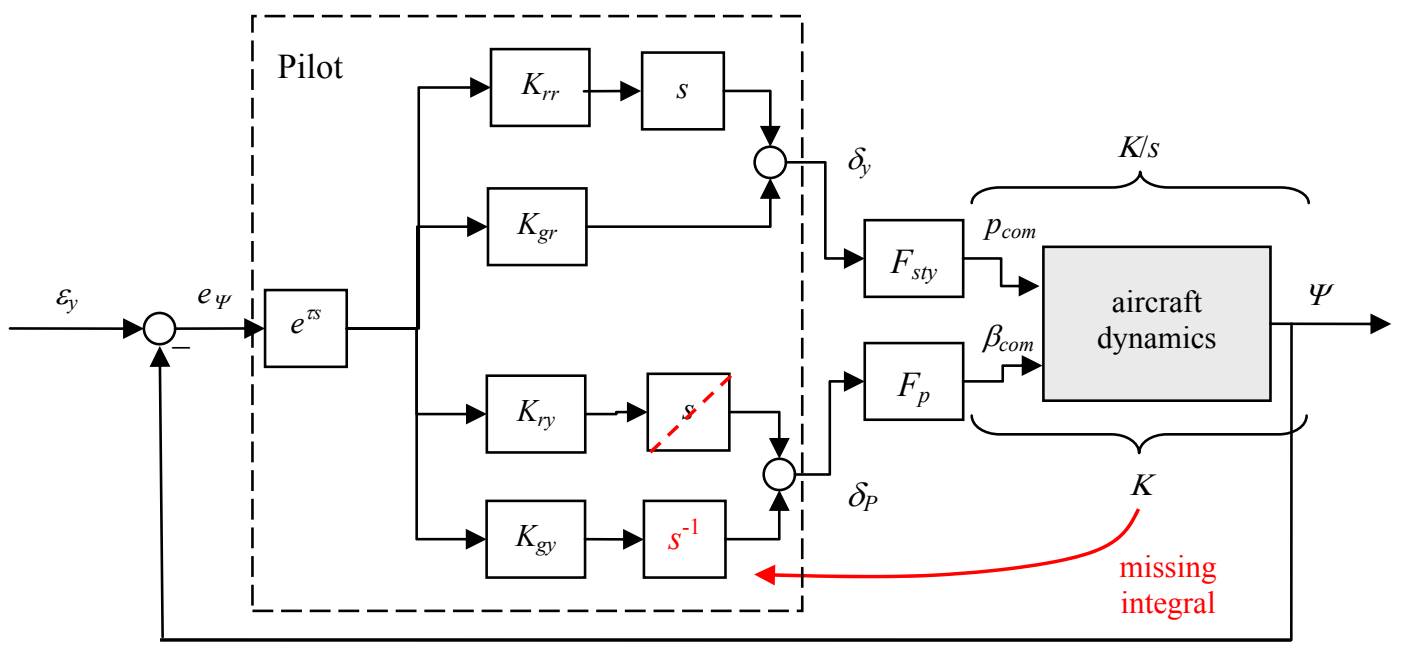

Figure 28. pilot model structure for the lateral-directional axis

The associated state equations are:

$$
\begin{aligned}
& \delta_{y}=\left(K_{g r} e_{\Psi}+K_{r r} \dot{e}_{\Psi}\right) e^{25} \\
& \dot{\delta}_{P}=\left(K_{g y} e_{\Psi}+K_{r y} \dot{e}_{\Psi}\right) e^{\sqrt{s}}
\end{aligned}
$$

Again, the elements $F_{P}$ and $F_{s t y}$ are included to describe the roll stick and pedal characteristics. The error $e_{\Psi}$ is again fed through a time delay $e^{\tau s}$, before being advanced to the two paths, generating the inputs for the rudder and the aileron. In analogy to the longitudinal pilot model the roll axis is again split into a proportional $K_{g r}$ and a parallel derivative path with an associated gain $K_{r r}$ acting on the errors' rate of change. Since the Tornado's numerator time constant $T_{\Phi 2}$ is rather high, leading to relatively slow flight path dynamics, the transfer behavior $p_{\text {com }} \rightarrow \Psi$ can be described by a $K / s$-approximation. Again, this leads to the $K / s$ crossover behavior, augmented with an additional derivative element, describing the pilot's efforts to control the rate of change of the error.
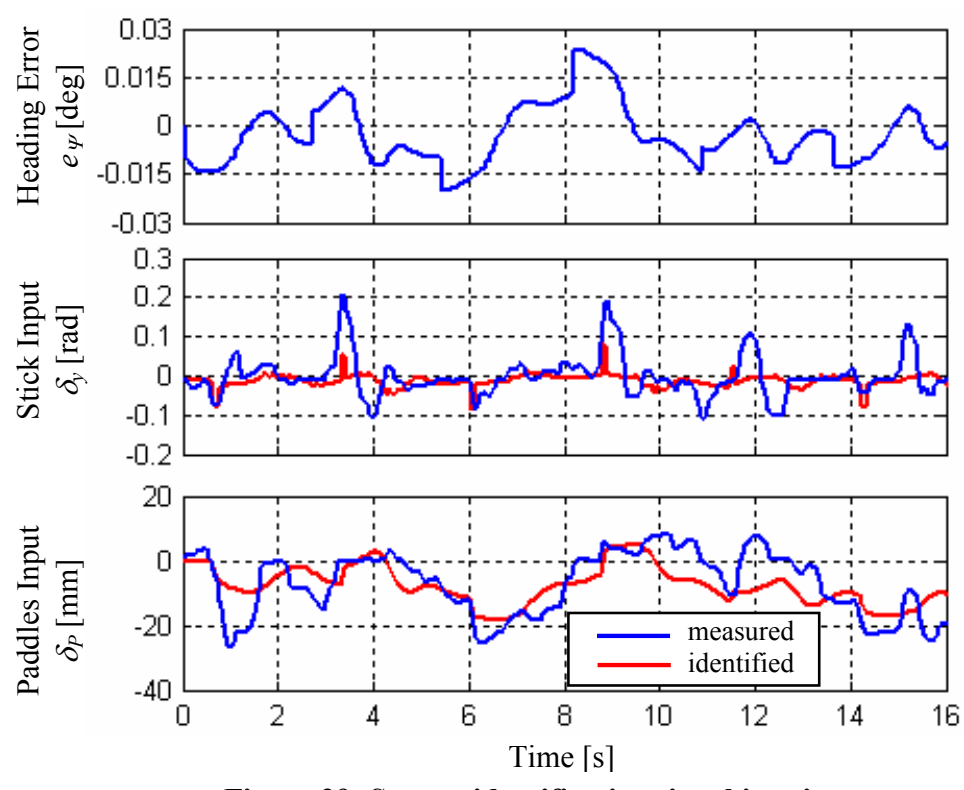

Figure 29: System identification time histories 
The lower path generates the pilot's rudder pedal inputs. The FCS features sideslip control $\left(\beta_{\text {com }}\right)$, leading to a proportional correlation between the pedal input and the aircraft heading, since a change in sideslip angle directly induces a heading change. In order to realize the crossover model transfer characteristics $(K / s)$ described in Ref. 2, an additional integration needs to be performed by the pilot, leading to the cancellation of the derivative in the rate path $\left(K_{r y}\right)$ and the addition of an integral block in the proportional path $\left(K_{g y}\right)$ as depicted in Fig. 28.

Figure 29 shows time histories of pilot inputs extracted from flight test data which are then compared to stick and pedal signals generated with the identified linear pilot model. Again, variable time delays and amplitude variations can be observed. This highlights the difficulties in the attempt to replicate a highly nonlinear system such as the human pilot by means of quasi-linear models, even for a very confined test setup with very small perturbations. In the given example the pilot uses sideslip and roll to the same extent to control the aircraft, making a correct identification even more difficult, due to considerable coupling effects. For cases, where pure sideslip control was used to minimize the lateral error $e_{\psi}$ significantly better system identification results could be achieved, which has been verified in practice.

\section{Conclusion}

The work described herein is regarded as a first step on the way to a more sophisticated pilot model for air-tosurface tracking. It was shown that the GRATE II system is an invaluable tool to investigate pilot dynamics in a realistic, operationally relevant environment, which cannot be achieved to the same degree when employing HUDbased generic tracking tasks. Further efforts will be made to refine the derived models to include combined, fully coupled dynamics, switching functions to address varying pilot control strategies and biomechanical aspects to account for interactions between the airframe dynamics and the physical properties of the human extremities used to manipulate the control devices.

\section{Acknowledgment}

The authors would like to thank the involved organisations for their extensive support. Without the close collaboration between the German Aerospace Center (DLR), the Bundeswehr Technical and Airworthiness Center for Aircraft (WTD 61) and the European Aeronautic Defence and Space Company (EADS) this work would not have been possible.

\section{References}

${ }^{1}$ Höhne, G., "Roll Ratcheting: Cause and Analysis," DLR-FB 2001-15,Braunschweig, 2001.

2 McRuer, D., "Mathematical Models of Human Pilot Behavior,"AGARD-AG-188, Neuillysur Seine, 1974.

${ }^{3}$ Hess, R. A., "A Structural Model of the Adaptive Human Pilot," AIAA Guidance, Navigation and Control Conference, AIAA-87-2523.

${ }^{4}$ Shafer, M.S., "Initial flight test of a ground deployed system for flying quality assessment," NASA Technical Memorandum 101700, Edwards, California, 1989.

5 Einsiedel, F., "GRATE II - Implementation of a ground deployed system for the evaluation of handling qualities," IB 1112007/49, Braunschweig/ Manching, 2007.

${ }^{6}$ Koehler, R., "GRATE - A new flight test tool for flying qualities evaluations," $73^{\text {rd }}$ Symposium on Flight Test Techniques, AGARD, 1988.

${ }^{7}$ Koehler, R., "Design and Implementation of Input Signals for Identification of Pilot/Aircraft Models," DFVLR-FB 84-08, Braunschweig, 1984.

${ }^{8}$ Ossmann, D., "Identification of a pilot model on the basis of flight test data for handling quality evaluation," Master Thesis, Technische Universität München, Institute of Flight System Dynamics, Munich, 2008.

9 Jategaonkar, R. V., Flight Vehicle System Identification: A Time Domain Mythology, AIAA, Reston, Virginia, 2006.

${ }^{10}$ Seher-Weiß, S., "User's Guide FITLAB Parameter Estimation Using MATLAB Version 2.0,” IB 111-2007/27, Braunschweig; 2007. 